\title{
ASYMPTOTIC ESTIMATES FOR THE $p$-LAPLACIAN ON INFINITE GRAPHS WITH DECAYING INITIAL DATA
}

\author{
DANIELE ANDREUCCI AND ANATOLI F. TEDEEV
}

\begin{abstract}
We consider the Cauchy problem for the evolutive discrete $p$-Laplacian in infinite graphs, with initial data decaying at infinity. We prove optimal sup and gradient bounds for nonnegative solutions, when the initial data has finite mass, and also sharp evaluation for the confinement of mass, i.e., the effective speed of propagation. We provide estimates for some moments of the solution, defined using the distance from a given vertex.

Our technique relies on suitable inequalities of Faber-Krahn type, and looks at the local theory of continuous nonlinear partial differential equations. As it is known, however, not all of this approach can have a direct counterpart in graphs. A basic tool here is a result connecting the supremum of the solution at a given positive time with the measure of its level sets at previous times.

We also consider the case of slowly decaying initial data, where the total mass is infinite.

This is a post-peer-review, pre-copyedit version of an article published in Potential Analysis 53 (2020), no. 2, 677-699. The final authenticated version is available online at: https://doi.org/10.1007/s11118019-09784-w
\end{abstract}

\section{INTRODUCTION}

We consider nonnegative solutions to the Cauchy problem for discrete degenerate parabolic equations

$$
\begin{aligned}
\frac{\partial u}{\partial t}(x, t)-\Delta_{p} u(x, t) & =0, & & x \in V, t>0, \\
u(x, 0) & =u_{0}(x) \geq 0, & & x \in V .
\end{aligned}
$$

Here $V$ is the set of vertices of the graph $G(V, E)$ with edge set $E \subset$ $V \times V$ and weight $\omega$, and

$$
\Delta_{p} u(x, t)=\frac{1}{d_{\omega}(x)} \sum_{y \in V}|u(y)-u(x)|^{p-2}(u(y)-u(x)) \omega(x, y) .
$$

The first author is member of the Gruppo Nazionale per la Fisica Matematica (GNFM) of the Istituto Nazionale di Alta Matematica (INdAM).

The second author was supported by Sapienza Grant C26V17KBT3. 
We assume that the graph $G$ is simple, undirected, infinite, connected with locally finite degree

$$
d_{\omega}(x)=\sum_{y \sim x} \omega(x, y),
$$

where we write $y \sim x$ if and only if $\{x, y\} \in E$. Here the weight $\omega: V \times V \rightarrow[0,+\infty)$ is symmetric, i.e., $\omega(x, y)=\omega(y, x)$, and is strictly positive if and only if $y \sim x$; then $\omega(x, x)=0$ for $x \in V$.

We assume also that $p>2$ and that $u_{0}$ is nonnegative; further assumptions on $u_{0}$ will be stated below.

We prove sharp sup bounds for large times of solutions corresponding to finite mass initial data; in order to prove the bound from below we find an optimal estimate for the effective speed of propagation of mass. We also determine the stabilization rate for data exhibiting slow decay 'at infinity', in a suitable sense. To the best of our knowledge such results are new in the framework of discrete nonlinear diffusion equations on graphs.

We apply an alternative approach, more local than the one in [23], [19] where the global arguments of semigroup theory are extended to graphs, actually in a more general setting which is out of the scope of this paper. We comment below in the Introduction on the inherent difficulty and even partial unfeasibility of a local approach in graphs. It is therefore an interesting and not trivial problem to understand how much of this body of techniques can be used in this environment. This paper can be seen as a cross section of this effort; specifically we look at the interplay between spread of mass and sup estimates, following ideas coming from the theory of continuous partial differential equations, with the differences required by the discrete character of graphs.

We recall the following notation: for any $R \in \boldsymbol{N}$, we let

$$
B_{R}\left(x_{0}\right)=\left\{x \in V \mid d\left(x, x_{0}\right) \leq R\right\} .
$$

Here $d$ is the standard combinatorial distance in $G$ so that $d$ only takes integral values. For any $f: V \rightarrow \boldsymbol{R}$ we set for all $q \geq 1, U \subset V$

$$
\begin{gathered}
\|f\|_{\ell^{q}(U)}^{q}=\sum_{x \in U}|f(x)|^{q} d_{\omega}(x), \quad\|f\|_{\ell^{\infty}(U)}=\sup _{x \in U}|f(x)|, \\
\mu_{\omega}(U)=\sum_{x \in U} d_{\omega}(x) .
\end{gathered}
$$

All the infinite sums in this paper are absolutely convergent. In the following we always assume, unless explicitly noted, that all balls are centered at a given fixed $x_{0} \in V$ and we write $B_{R}\left(x_{0}\right)=B_{R}$. We denote generic constants depending on the parameters of the problem 
by $\gamma$ (large constants), $\gamma_{0}$ (small constants). We also set for all $A \subset V$

$$
\chi_{A}(x)=1, \quad x \in A ; \quad \chi_{A}(x)=0, \quad x \notin A .
$$

Definition 1.1. We say that $G$ satisfies a global Faber-Krahn inequality for a given $p>1$ and function $\Lambda_{p}:(0,+\infty) \rightarrow(0,+\infty)$ if for any $v>0$ and any finite subset $U \subset V$ with $\mu_{\omega}(U)=v$ we have

$$
\Lambda_{p}(v) \sum_{x \in U}|f(x)|^{p} d_{\omega}(x) \leq \sum_{x, y \in(U)_{1}}|f(y)-f(x)|^{p} \omega(x, y),
$$

for all $f: V \rightarrow \boldsymbol{R}$ such that $f(x)=0$ if $x \notin U$; here

$$
(U)_{1}=\{x \in V \mid d(x, U) \leq 1\} .
$$

We assume throughout that $\Lambda_{p} \in C(0,+\infty)$ is decreasing and that two suitable positive constants $N, \omega$ exists such that

$$
\begin{aligned}
& v \mapsto \Lambda_{p}(v)^{-1} v^{-\frac{p}{N}}, \quad v>0, \quad \text { is nondecreasing; } \\
& v \mapsto \Lambda_{p}(v)^{-1} v^{-\omega}, \quad v>0, \quad \text { is nonincreasing. }
\end{aligned}
$$

An important class of functions in our approach is given by

$$
\psi_{r}(s)=s^{\frac{p-2}{r}} \Lambda_{p}\left(s^{-1}\right), \quad s>0,
$$

for each fixed $r \geq 1$. They, or more exactly their inverses, give the correct time-space scaling for the equation (1.1), see for example Theorems 1.9 and 1.11 below.

If we make the additional assumption that for some constant $c>0$

$$
\Lambda_{p}(v) \geq c \mathcal{R}(v)^{-p}, \quad v>0,
$$

where $\mathcal{R}:(0,+\infty) \rightarrow(0,+\infty)$ is such that $\mu_{\omega}\left(B_{\mathcal{R}(v)}\right)=v$, we may connect $\psi_{1}$ to the measure of a ball in $G$. This in turn allows us to prove sharpness of our $\ell^{1}-\ell^{\infty}$ estimate. Property (1.7) is rather natural. For instance it is known to hold for the explicit examples in Subsection 1.1, to which we refer for implementations of our results in some concrete relevant cases.

Remark 1.2. The constant $N$ in (1.4) has no intrinsic meaning in this paper, and it is employed here only with the purpose of making easier the comparison with the case of standard regular graphs $\boldsymbol{Z}^{N}$, where $\Lambda_{p}(v)=\gamma_{0} v^{-p / N}$, see Subsection 1.1.

Remark 1.3. Let $x \in V$ and choose $U=\{x\}, f=\chi_{U}$ in (1.3), which then yields

$$
\Lambda_{p}\left(d_{\omega}(x)\right) d_{\omega}(x) \leq 2 d_{\omega}(x) .
$$


Since $\Lambda_{p}$ is decreasing by assumption we infer

$$
d_{\omega}(x) \geq \Lambda_{p}^{(-1)}(2) .
$$

In the terminology of [19] then $d_{\omega}$ is non-degenerate, and $G$ is uniformly locally finite.

A further remark in connection with (1.9) is perhaps in order: clearly according to its definition the Faber-Krahn function $\Lambda_{p}(v)$ is defined for uniformly positive $v$ according to (1.9), so that (1.4), (1.5) should be assumed for such $v$. Aiming at a technically streamlined framework, we extend $\Lambda_{p}$ to all $v>0$, while easily preserving the latter assumptions. However one can check that for large times, $\Lambda_{p}$ is evaluated at large arguments in our results, which are thus independent of this extension.

Remark 1.4. A consequence of (1.9) is that any bound in $\ell^{q}(V)$ yields immediately a uniform pointwise bound: if $v \in \ell^{q}(V)$,

$$
|v(z)|^{q} \leq|v(z)|^{q} \frac{d_{\omega}(z)}{\Lambda_{p}^{(-1)}(2)} \leq \frac{1}{\Lambda_{p}^{(-1)}(2)}\|v\|_{\ell^{q}(V)}^{q}, \quad z \in V .
$$

In turn this implies that $\ell^{p}(V) \subset \ell^{q}(V)$ if $p<q$, since

$$
\sum_{x \in V}|f(x)|^{q} d_{\omega}(x) \leq M^{q-p} \sum_{x \in V}|f(x)|^{p} d_{\omega}(x),
$$

for a suitable $M$ as in (1.10).

Definition 1.5. We say that $u \in L^{\infty}\left(0, T ; \ell^{r}(V)\right)$ is a solution to (1.1) if $u(x) \in C^{1}([0, T])$ for every $x \in V$ and $u$ satisfies (1.1) in the classical pointwise sense.

A solution to (1.1)-(1.2) also is required to take the initial data prescribed by (1.2), for each $x \in V$.

We refer the reader to [19] for existence and uniqueness of solutions. To make this paper more self-contained however we sketch in Section 2 a proof of existence in Proposition 2.6 (in $\ell^{q}, q>1$, see Theorem 1.9 for $q=1$ ). Uniqueness of solutions follows via comparison on appealing to Proposition 2.7, known since [23].

Our first two results are typical of the local approach we pursue. All solutions we consider below are nonnegative.

Proposition 1.6. Let $u: V \rightarrow \boldsymbol{R}$ be a solution to (1.1), with $u \in$ $L^{\infty}\left(0, T ; \ell^{r}(V)\right)$ for some $r \geq 1$. Then for all $x \in V, 0<t<T$

$$
u(x, t) \leq k,
$$


provided $k>0$ satisfies for a suitable $\gamma_{0}(p, N)$

$$
k^{-1} t^{-\frac{1}{p-2}} \Lambda_{p}\left(\sup _{\frac{t}{4}<\tau<t} \mu_{\omega}(\{x \in V \mid u(x, \tau)>k / 2\})\right)^{-\frac{1}{p-2}} \leq \gamma_{0} .
$$

Corollary 1.7. Under the assumptions in Proposition 1.6, we have

$$
u(x, t) \leq \gamma \sup _{0<\tau<t}\|u(\tau)\|_{\ell^{r}(V)}\left[\psi_{r}^{(-1)}\left(t^{-1} \sup _{0<\tau<t}\|u(\tau)\|_{\ell^{r}(V)}^{-(p-2)}\right)\right]^{\frac{1}{r}},
$$

for all $x \in V, 0<t<T$. Here $\psi_{r}^{(-1)}$ is the inverse function of $\psi_{r}$ as defined in (1.6).

Remark 1.8. One can check easily using the fact that $\Lambda_{p}$ is nonincreasing that

$$
a \mapsto a \psi_{r}^{(-1)}\left(s a^{-(p-2)}\right)^{\frac{1}{r}}
$$

is nondecreasing in $a>0$ for each fixed $s>0$.

Next Theorem follows directly from the estimates we stated above. Note that conservation of mass in (1.14) was proved also in [19], while the other estimates are new, as far as we know.

We use from now on for $f: V \rightarrow \boldsymbol{R}$ the notation

$$
D_{y} f(x)=f(y)-f(x)=-D_{x} f(y), \quad x, y \in V .
$$

Theorem 1.9. Let $u_{0} \in \ell^{1}(V), u_{0} \geq 0$. Then problem (1.1)-(1.2) has a unique solution satisfying for all $t>0$

$$
\begin{aligned}
\|u(t)\|_{\ell^{1}(V)} & =\left\|u_{0}\right\|_{\ell^{1}(V)}, \\
\|u(t)\|_{\ell^{\infty}(V)} & \leq \gamma\left\|u_{0}\right\|_{\ell^{1}(V)} \psi_{1}^{(-1)}\left(t^{-1}\left\|u_{0}\right\|_{\ell^{1}(V)}^{-(p-2)}\right) .
\end{aligned}
$$

In addition u satisfies

$$
\begin{aligned}
& \int_{0}^{t} \sum_{x, y \in V}\left|D_{y} u(x, \tau)\right|^{p-1} \omega(x, y) \mathrm{d} \tau \\
& \leq \gamma t^{\frac{1}{p}}\left\|u_{0}\right\|_{\ell^{1}(V)}^{\frac{2(p-1)}{p}} \psi_{1}^{(-1)}\left(t^{-1}\left\|u_{0}\right\|_{\ell^{1}(V)}^{-(p-2)}\right)^{\frac{p-2}{p}} .
\end{aligned}
$$

Remark 1.10. We notice that one could exploit (1.14), (1.15) to derive trivially a bound of the integral in (1.16). This is due of course to the fact that the $p$-laplacian in our setting is discrete, and it would not be possible in the framework of continuous partial differential equations.

Such a bound however is not sharp, and for example could not be used in the proof of Theorem 1.11.

In other instances where optimality is not needed we exploit a device similar to the one just described, relying on Remark 1.4; see for example the proof of Lemma 2.4. 
So far our extension to graphs of methods and results of continuous differential equations has been successful. However, in the latter setting a standard device to prove optimality of the bound in (1.15) relies on the property of finite speed of propagation (i.e., solutions with initially bounded support keep this feature for all $t>0$ ). In the setting of graphs this property strikingly fails, as shown in [19]. As a technical but perhaps worthwile side remark, we note that all the main ingredients in the proof of finite speed of propagation (see [5], [6]) seem to be available in graphs too: embeddings as in [24], Caccioppoli inequalities as in Lemma 2.2 below, and of course iterative techniques as the one displayed in the proof of Proposition 1.6. The key exception in this regard is the fact that full localization via an infinite sequence of nested shrinking balls is clearly prohibited by the discrete metric at hand. This is a point of marked difference with the continuous setting.

Still we can prove sharpness of our $\ell^{1}-\ell^{\infty}$ bound (1.15) by means of the following result of confinement of mass. By the same argument we can estimate also a suitable moment of the solution, which is also a new result for nonlinear diffusion in graphs, see Section 5.

Theorem 1.11. Let $u_{0} \geq 0$ be finitely supported. Then for every $1>\varepsilon>0$ there exists a $\Gamma>0$ such that

$$
\|u(t)\|_{\ell^{1}\left(B_{R}\right)} \geq(1-\varepsilon)\left\|u_{0}\right\|_{\ell^{1}(V)}, \quad t>0
$$

provided $B_{\lfloor R / 2\rfloor}$ contains the support of $u_{0}$, and $R$ is chosen so that

$$
R \geq \Gamma t^{\frac{1}{p}}\left\|u_{0}\right\|_{\ell^{1}(V)}^{\frac{p-2}{p}} \psi_{1}^{(-1)}\left(t^{-1}\left\|u_{0}\right\|_{\ell^{1}(V)}^{-(p-2)}\right)^{\frac{p-2}{p}} \geq 8 .
$$

In addition, provided $R$ is chosen as in (1.18), for $\varepsilon=1 / 2$, and $\alpha \in$ $(0,1)$,

$$
\sum_{x \in V} d\left(x, x_{0}\right)^{\alpha} u(x, t) d_{\omega}(x) \leq \gamma R^{\alpha}\left\|u_{0}\right\|_{\ell^{1}(V)}, \quad t>0 .
$$

Next we exploit the estimate (1.17)-(1.18) in order to show that up to a change in the constant we can reverse the inequality in (1.15), proving at once the optimality of both results.

Corollary 1.12. Under the assumptions in Theorem 1.11, let in addition $\Lambda_{p}$ satisfy (1.7). Then

$$
\|u(t)\|_{\ell^{\infty}(V)} \geq \frac{\left\|u_{0}\right\|_{\ell^{1}(V)}}{2 \mu_{\omega}\left(B_{R}\right)} \geq \gamma_{0}\left\|u_{0}\right\|_{\ell^{1}(V)} \psi_{1}^{(-1)}\left(t^{-1}\left\|u_{0}\right\|_{\ell^{1}(V)}^{-(p-2)}\right),
$$

where $R$ is as in (1.18), for $\varepsilon=1 / 2$.

Clearly, owing to the comparison principle of Proposition 2.7, results like those in (1.17) and (1.20) may be proved even dropping the 
assumption that $u_{0}$ is finitely supported; for the sake of brevity we omit the details.

In order to state our last result we need to introduce the following function, which essentially gives the correct scaling between time and space in the case of slow decay initial data: for $u_{0} \in \ell^{q}(V) \backslash \ell^{1}(V)$ for some $q>1$ set

$$
T_{u_{0}}\left(R, x_{0}\right)=\left[\frac{\left\|u_{0}\right\|_{\ell^{1}\left(B_{R}\left(x_{0}\right)\right)}}{\left\|u_{0}\right\|_{\ell^{q}\left(V \backslash B_{R}\left(x_{0}\right)\right)}^{q}}\right]^{\frac{p-2}{q-1}} \Lambda_{p}\left(\left(\frac{\left\|u_{0}\right\|_{\ell^{1}\left(B_{R}\left(x_{0}\right)\right)}}{\left\|u_{0}\right\|_{\ell^{q}\left(V \backslash B_{R}\left(x_{0}\right)\right)}}\right)^{\frac{q}{q-1}}\right)^{-1}
$$

for $R \in \boldsymbol{N}, x_{0} \in V$. Clearly for each fixed $x_{0}$ the function $T_{u_{0}}$ is nondecreasing in $R$ and $T_{u_{0}}\left(R, x_{0}\right) \rightarrow+\infty$ as $R \rightarrow \infty$. Conversely, $T_{u_{0}}\left(0, x_{0}\right)$ may be positive. However it can be easily seen that for any given $\varepsilon>0$ there exists $x_{0}$ such that $T_{u_{0}}\left(0, x_{0}\right)<\varepsilon$.

Theorem 1.13. Let $u_{0} \in \ell^{q}(V) \backslash \ell^{1}(V)$ for some $q>1$. Then for all $t>0, x_{0} \in V$

$$
\|u(t)\|_{\ell^{\infty}(V)} \leq \gamma\left\|u_{0}\right\|_{\ell^{1}\left(B_{R}\left(x_{0}\right)\right)} \psi_{1}^{(-1)}\left(t^{-1}\left\|u_{0}\right\|_{\ell^{1}\left(B_{R}\left(x_{0}\right)\right)}^{-(p-2)}\right),
$$

provided $R$ is chosen so that

$$
t \leq T_{u_{0}}\left(R, x_{0}\right),
$$

the optimal choice being of course the minimum $R=R(t)$ such that (1.23) holds true.

Let us comment briefly on the existing literature on the non-linear p-Laplacian in graphs. The papers [23], [19], deal with the Cauchy problem applying techniques inspired from the theory of semigroups of continuous differential operators. They consider a more general variety of weighted graphs and operators than we do here, dealing e.g., with existence, uniqueness, time regularity, possible extinction in a finite time. However our results do not seem to be easily reached by this approach. We also quote [20] where a connection between Cheeger constants and the eigenvalues of the $p$-laplacian is drawn in a very flexible setting.

Boundary problems on finite subgraphs are also considered in several papers dealing with features like blow up or extinction; we quote only [12] and [13].

The case of the discrete linear Laplacian where $p=2$ is more classical, also for its connections with probability theory (see e.g., [2] and references therein), and is often attacked by means of suitable parallels with the theory of heat kernels in manifolds. We quote [14], [9] where a connection is drawn between properties of heat kernels, of graphs and 
Faber-Krahn functions.

In [22] heat kernels are used to study the blow up of solutions to the Cauchy problem for a semilinear equation on a possibly infinite graph.

The subject of diffusion in graphs is popular also owing to its applicative interest. We refer the reader to [23], [17] and to the references therein for more on this point.

Finally we recall the papers [8], [24] and books [11], [18] for basic information on functional analysis on graphs and manifolds.

We mention that in our setting it is still valid the argument in [10] showing that optimal decay rates imply suitable embeddings.

Here we look essentially at the approach of [16] and [7].

The paper is organized as follows: Section 2 is devoted to preliminary material. Proposition 1.6 and its Corollary 1.7 are proved in Section 3, while Section 4 contains the proof of Theorem 1.9 and Section 5 deals with Theorem 1.11 and Corollary 1.12. Finally Theorem 1.13 is proved in Section 6.

1.1. Examples. 1) As a first example we consider the case of the standard lattice $G=Z^{N}$, where one can take $\Lambda_{p}(v)=\gamma_{0} v^{-p / N}$, according to the results of [26], [24]. This is the case where comparison with the Cauchy problem for the continuous $p$-Laplacian is more straightforward. In this case

$$
\psi_{r}(s)=\gamma_{0} s^{\frac{N(p-2)+p r}{N r}}, \quad s>0,
$$

and for example estimate (1.15) becomes

$$
\|u(t)\|_{\ell^{\infty}(V)} \leq \gamma\left\|u_{0}\right\|_{\ell^{1}(V)}^{\frac{p}{N-2)+p}} t^{-\frac{N}{N(p-2)+p}}
$$

while the critical radius for expansion of mass in (1.18) amounts to

$$
R \geq \gamma\left\|u_{0}\right\|_{\ell^{1}(V)}^{\frac{p-2}{N(p-2)+p}} t^{\frac{1}{N(p-2)+p}} .
$$

We remark that both results formally coincide with the corresponding ones for the continuous $p$-Laplacian in $\boldsymbol{R}^{N}$, see [16].

Next we apply Theorem 1.13 to the following initial data: for $x=$ $\left(x_{1}, \ldots, x_{N}\right) \in Z^{N}$ set $u_{0}(x)=\left(\left|x_{1}\right|+\cdots+\left|x_{N}\right|\right)^{-\alpha}$ for a given $0<$ $\alpha<N$. Let us write here $a(s) \simeq b(s)$ if $\gamma_{0} a(s) \leq b(s) \leq \gamma a(s)$ for two constants independent of $s$. One can see that

$$
\left\|u_{0}\right\|_{\ell^{1}\left(B_{R}(0)\right)} \simeq R^{N-\alpha} ; \quad\left\|u_{0}\right\|_{\ell^{q}\left(V \backslash B_{R}(0)\right)} \simeq R^{N-\alpha q},
$$

for all $q>N / \alpha$. Therefore in this case

$$
T_{u_{0}}(R, 0) \underset{8}{\simeq} R^{\alpha(p-2)+p}
$$


and the estimate in (1.22) essentially amounts to the decay rate $t^{-\alpha /(\alpha(p-2)+p)}$, which is the expected one in view of the results of [25].

2) One can treat also other examples of product graphs; for instance if $H$ is a finite connected graph we let $G=H \times Z^{N}$ and recover results similar to the ones of the previous example.

3) All examples where the Faber-Krahn function is estimated for $p=2$ yield also examples in our case of $p>2$, as it follows from applying Hölder's inequality; see e.g., [14], [9].

\section{Preliminary material}

\subsection{Caccioppoli type inequalities.}

Lemma 2.1. Let $q>0, p>2, h \geq 0, u, v: V \rightarrow \boldsymbol{R}$. Then for all $x$, $y \in V$

$$
\begin{gathered}
\left(\left|D_{y} u(x)\right|^{p-2} D_{y} u(x)-\left|D_{y} v(x)\right|^{p-2} D_{y} v(x)\right) D_{y}(u(x)-v(x)-h)_{+}^{q} \\
\geq \gamma_{0}\left|D_{y}(u(x)-v(x)-h)_{+}^{\frac{q-1+p}{p}}\right|^{p} \cdot
\end{gathered}
$$

Proof. First we remark that we may assume $h=0$, by renaming $\tilde{v}=$ $v+h$. The corresponding version of (2.1) clearly holds true if $D_{y} u(x)=$ $D_{y} v(x)$.

If $D_{y} u(x) \neq D_{y} v(x)$ the left hand side of (2.1) with $h=0$ can be written as, on appealing also to a classical elementary result in monotone operators, see [15],

$$
\begin{array}{r}
\left(\left|D_{y} u(x)\right|^{p-2} D_{y} u(x)-\left|D_{y} v(x)\right|^{p-2} D_{y} v(x)\right) D_{y}(u(x)-v(x)) \mathcal{A} \\
\geq \gamma_{0}(p)\left|D_{y}(u(x)-v(x))\right|^{p} \mathcal{A},
\end{array}
$$

where we define

$$
\mathcal{A}=\frac{D_{y}(u(x)-v(x))_{+}^{q}}{D_{y}(u(x)-v(x))} \geq 0 .
$$

On the other hand, we write the right hand side of $(2.1)$ with $h=0$ as

$$
\left|D_{y}(u(x)-v(x))\right|^{p} \mathcal{B}, \quad \mathcal{B}:=\left|\frac{D_{y}(u(x)-v(x))_{+}^{\frac{q-1+p}{p}}}{D_{y}(u(x)-v(x))}\right|^{p} .
$$

Therefore we have only to prove that $\mathcal{A} \geq \gamma_{0} \mathcal{B}$. Clearly in doing so we may assume without loss of generality that

$$
u(y)-v(y)>\underset{9}{u} u(x)-v(x) .
$$


Hence it is left to prove that

$$
\begin{aligned}
{[u(y)-v(y)} & -(u(x)-v(x))]^{p-1}\left[(u(y)-v(y))_{+}^{q}-(u(x)-v(x))_{+}^{q}\right] \\
& \geq \gamma_{0}\left[(u(y)-v(y))_{+}{ }^{\frac{q-1+p}{p}}-(u(x)-v(x))_{+}^{\frac{q-1+p}{p}}\right]^{p} \cdot
\end{aligned}
$$

Denote

$$
a=u(y)-v(y), \quad b=u(x)-v(x) .
$$

If $b \leq 0,(2.4)$ is obviously satisfied with $\gamma_{0}=1$. If $b>0$, by Hölder's inequality we have

$$
\begin{aligned}
& {\left[a^{\frac{q-1+p}{p}}-b^{\frac{q-1+p}{p}}\right]^{p}=\left[\frac{q-1+p}{p} \int_{b}^{a} s^{\frac{q-1}{p}} \mathrm{~d} s\right]^{p}} \\
& \leq \gamma(q, p)\left[\int_{b}^{a} s^{q-1} \mathrm{~d} s\right]\left[\int_{b}^{a} \mathrm{~d} s\right]^{p-1} \leq \gamma(q, p)\left(a^{q}-b^{q}\right)(a-b)^{p-1},
\end{aligned}
$$

proving (2.4) and concluding the proof.

In the following all radii of balls in $G$ will be assumed to be natural numbers. Let $R_{2} \geq R_{1}+1, R_{1}, R_{2}>0$; we define the cutoff function $\zeta$ in $B_{R_{2}}\left(x_{0}\right)$ by means of

$$
\begin{array}{llrl}
\zeta(x)=1, & & x \in B_{R_{1}}\left(x_{0}\right), \\
\zeta(x)=\frac{R_{2}-d\left(x, x_{0}\right)}{R_{2}-R_{1}}, & & x \in B_{R_{2}} \backslash B_{R_{1}}\left(x_{0}\right), \\
\zeta(x)=0, & & x \notin B_{R_{2}}\left(x_{0}\right) .
\end{array}
$$

The function $\zeta$ is chosen so that

$$
\left|D_{y} \zeta(x)\right|=|\zeta(y)-\zeta(x)| \leq \frac{1}{R_{2}-R_{1}}, \quad x \sim y .
$$

For $\tau_{1}>\tau_{2}>0$ we also define the standard nonnegative cutoff function $\eta \in C^{1}(\boldsymbol{R})$ such that

$$
\eta(t)=0, t \geq \tau_{1} ; \quad \eta(t)=0, t \leq \tau_{2} ; \quad 0 \leq \eta^{\prime}(t) \leq \frac{2}{\tau_{1}-\tau_{2}}, t \in \boldsymbol{R} .
$$

Our next Lemma is not used in the sequel; we present it here to substantiate our claim made in the Introduction that suitable local Caccioppoli type inequalities are available in the nonlinear setting, and also for its possible independent interest. The proof is somehow more complex than in the continuous case. 
Lemma 2.2. Let $u$ be a solution of (1.1) in $V \times(0, T), x_{0} \in V$. Then for $T>\tau_{1}>\tau_{2}>0, R_{2}>R_{1}+1, R_{1}>0, h>k>0,1>\theta>0$ we have

$$
\begin{aligned}
& \sup _{\tau_{1}<\tau<t} \sum_{x \in B_{R_{1}}\left(x_{0}\right)}(u(x, \tau)-h)_{+}^{\theta+1} \zeta(x)^{p} d_{\omega}(x) \\
& \quad+\int_{\tau_{1}}^{t} \sum_{x \in B_{R_{1}}\left(x_{0}\right), y \in V}\left|D_{y}(u(x, \tau)-h)_{+}^{\frac{p+\theta-1}{p}}\right|^{p} \omega(x, y) \mathrm{d} \tau \\
& \quad \leq \frac{\gamma}{\tau_{1}-\tau_{2}} \int_{\tau_{2}}^{t} \sum_{x \in B_{R_{2}}\left(x_{0}\right)}(u(x, \tau)-h)_{+}^{\theta+1} d_{\omega}(x) \mathrm{d} \tau+\gamma A^{\frac{1}{p}} B^{\frac{p-1}{p}}+\gamma A,
\end{aligned}
$$

where

$$
\begin{aligned}
& A=\frac{1}{\left(R_{2}-R_{1}\right)^{p}} \int_{\tau_{2}}^{t} \sum_{x \in B_{R_{2}}\left(x_{0}\right)}(u(x, \tau)-k)_{+}^{p+\theta-1} d_{\omega}(x) \mathrm{d} \tau, \\
& B=h^{p}(h-k)^{\theta-1} \int_{\tau_{2}}^{t} \mu_{\omega}\left(B_{R_{2}}\left(x_{0}\right) \cap\{2 h \geq u(x, \tau)>h\}\right) \mathrm{d} \tau .
\end{aligned}
$$

Remark 2.3. The term $A^{1 / p} B^{(p-1) / p}$ in (2.6) can be reduced to one containing only $A$ by means of Young's and Chebychev's inequalities.

Proof. We multiply (1.1) against $\zeta(x)^{p} \eta(t)^{p}(u(x, t)-h)_{+}^{\theta}$ and apply the well known formula of integration by parts

$$
\begin{aligned}
\sum_{x, y \in V}\left|D_{y} u(x)\right|^{p-2} D_{y} u(x) f(x) \omega(x, y) & \\
& =-\frac{1}{2} \sum_{x, y \in V}\left|D_{y} u(x)\right|^{p-2} D_{y} u(x) D_{y} f(x) \omega(x, y),
\end{aligned}
$$

where $f: V \rightarrow \boldsymbol{R}$ has finite support. Below we denote $B_{R}\left(x_{0}\right)=B_{R}$ for simplicity of notation. 
We obtain

$$
\begin{aligned}
& J_{1}+J_{2}:=\frac{1}{\theta+1} \sum_{x \in B_{R_{2}}}(u(x, t)-h)_{+}^{\theta+1} \zeta(x)^{p} \eta(t)^{p} d_{\omega}(x) \\
& +\frac{1}{2} \int_{0}^{t} \sum_{x, y \in V}\left|D_{y} u(x, \tau)\right|^{p-2} D_{y} u(x, \tau) D_{y}\left[(u(x, \tau)-h)_{+}^{\theta} \zeta(x)^{p}\right] \omega(x, y) \eta(\tau)^{p} \mathrm{~d} \tau \\
& =\frac{p}{\theta+1} \int_{0}^{t} \sum_{x \in B_{R_{2}}}(u(x, \tau)-h)_{+}^{\theta+1} \zeta(x)^{p} \eta(\tau)^{p-1} \eta^{\prime}(\tau) d_{\omega}(x) \mathrm{d} \tau=: J_{3} .
\end{aligned}
$$

We split $J_{2}$ according to the equality

$$
D_{y}\left[(u(x, \tau)-h)_{+}^{\theta} \zeta(x)^{p}\right]=\zeta(y)^{p} D_{y}(u(x, \tau)-h)_{+}^{\theta}+(u(x, \tau)-h)_{+}^{\theta} D_{y} \zeta(x)^{p} .
$$

Next we appeal to Lemma 2.1 with $v=0$ to get

$$
\left|D_{y} u(x, \tau)\right|^{p-2} D_{y} u(x, \tau) D_{y}\left[(u(x, \tau)-h)_{+}^{\theta}\right] \geq \gamma_{0}\left|D_{y}(u(x, \tau)-h)_{+}^{\frac{p+\theta-1}{p}}\right|^{p} .
$$

Thus from (2.7) we infer the bound

$$
J_{1}+J_{21}+J_{22} \leq J_{3}+J_{23}
$$

where

$$
\begin{aligned}
& J_{21}=\gamma_{0} \int_{0}^{t} \sum_{x, y \in V}\left|D_{y}(u(x, \tau)-h)_{+}^{\frac{p+\theta-1}{p}}\right|^{p} \zeta(y)^{p} \omega(x, y) \eta(\tau)^{p} \mathrm{~d} \tau, \\
& J_{22}=\frac{1}{4} \int_{0}^{t} \sum_{x, y \in V}\left|D_{y} u(x, \tau)\right|^{p-2} D_{y} u(x, \tau) D_{y}\left[(u(x, \tau)-h)_{+}^{\theta}\right] \zeta(y)^{p} \omega(x, y) \eta(\tau)^{p} \mathrm{~d} \tau, \\
& J_{23}=\frac{1}{2} \int_{0}^{t} \sum_{x, y \in V}\left|D_{y} u(x, \tau)\right|^{p-1}\left|D_{y} \zeta(x)^{p}\right|(u(x, \tau)-h)_{+}^{\theta} \eta(\tau)^{p} \omega(x, y) \mathrm{d} \tau .
\end{aligned}
$$

The reason to preserve the fraction $J_{22}$ of $J_{2}$ (rather than treating it as in $J_{21}$ ) will become apparent presently. Let us introduce the functions

$$
\begin{gathered}
H(x, y ; r)=\max \left[(u(x, \tau)-r)_{+},(u(y, \tau)-r)_{+}\right], \\
\chi_{x, y}=1, \quad \text { if } H(x, y ; h)>0 ; \quad \chi_{x, y}=0, \quad \text { if } H(x, y ; h)=0 .
\end{gathered}
$$

Note that $r>0$ is arbitrary in the definition of $H$ but we fix $r=h$ in the definition of $\chi_{x, y}$. Next we select $0<k<h$; by elementary 
calculations and Young's inequality we get

$$
\begin{aligned}
J_{23} \leq & \frac{p}{2} \int_{0}^{t} \sum_{x, y \in V}\left|D_{y} u(x, \tau)\right|^{p-1}\left|D_{y} \zeta(x)\right|(\zeta(x)+\zeta(y))^{p-1} H(x, y ; k)^{\theta} \chi_{x, y} \omega(x, y) \eta(\tau)^{p} \mathrm{~d} \tau \\
\leq & \varepsilon \int_{0}^{t} \sum_{x, y \in V}\left|D_{y} u(x, \tau)\right|^{p}\left(\zeta(x)^{p}+\zeta(y)^{p}\right) H(x, y ; k)^{\theta-1} \chi_{x, y} \omega(x, y) \eta(\tau)^{p} \mathrm{~d} \tau \\
& +\gamma \varepsilon^{1-p} \int_{0}^{t} \sum_{x, y \in V}\left|D_{y} \zeta(x)\right|^{p} H(x, y ; k)^{p+\theta-1} \omega(x, y) \eta(\tau)^{p} \mathrm{~d} \tau=: J_{231}+J_{232} .
\end{aligned}
$$

We want to absorb partially the term $J_{231}$ into $J_{22}$, for a suitable choice of $\varepsilon$. To this end we observe that by a change of variables we have

$$
\begin{aligned}
J_{22} & =\frac{1}{4} \int_{0}^{t} \sum_{x, y \in V}\left|D_{x} u(y, \tau)\right|^{p-1}\left|D_{x}(u(y, \tau)-h)_{+}^{\theta}\right| \zeta(x)^{p} \omega(y, x) \eta(\tau)^{p} \mathrm{~d} \tau \\
& =\frac{1}{4} \int_{0}^{t} \sum_{x, y \in V}\left|D_{y} u(x, \tau)\right|^{p-1}\left|D_{y}(u(x, \tau)-h)_{+}^{\theta}\right| \zeta(x)^{p} \omega(x, y) \eta(\tau)^{p} \mathrm{~d} \tau \\
& =\frac{1}{8} \int_{0}^{t} \sum_{x, y \in V}\left|D_{y} u(x, \tau)\right|^{p-1}\left|D_{y}(u(x, \tau)-h)_{+}^{\theta}\right|\left(\zeta(x)^{p}+\zeta(y)^{p}\right) \omega(x, y) \eta(\tau)^{p} \mathrm{~d} \tau .
\end{aligned}
$$

Then by elementary calculus

$$
\begin{aligned}
\chi_{x, y}\left|D_{y}(u(x, \tau)-h)_{+}^{\theta}\right| & \geq \chi_{x, y} \theta\left|D_{y}(u(x, \tau)-h)_{+}\right| H(x, y ; h)^{\theta-1} \\
& \geq \chi_{x, y} \theta\left|D_{y}(u(x, \tau)-h)_{+}\right| H(x, y ; k)^{\theta-1} .
\end{aligned}
$$

Next we discriminate three cases in (2.10), aggregating equivalent symmetric cases: i) $u(x, \tau)>h, u(y, \tau)>h$. In this case clearly

$$
\left|D_{y}(u(x, \tau)-h)_{+}\right|=\left|D_{y} u(x, \tau)\right| \text {. }
$$

ii) $u(x, \tau)>2 h, h \geq u(y, \tau)$. Then

$$
\left|D_{y}(u(x, \tau)-h)_{+}\right| \geq \frac{u(x, \tau)}{2} \geq \frac{1}{2}\left|D_{y} u(x, \tau)\right| .
$$

iii) $2 h \geq u(x, \tau)>h \geq u(y, \tau)$. In this case $J_{22}$ does not offer any help.

We rather bound directly this part of $J_{231}$ as shown below.

Collecting the estimates above we see that, provided $\varepsilon \leq 1 / 16$,

$$
J_{231} \leq J_{22}+\varepsilon 2^{p+2} h^{p}(h-k)^{\theta-1} \int_{\tau_{2}}^{t} \mu_{\omega}\left(B_{R_{2}} \cap\{2 h \geq u(x, \tau)>h\}\right) \mathrm{d} \tau .
$$


Hence we have transformed (2.9) into

$$
J_{1}+J_{21} \leq J_{3}+\gamma \varepsilon B+\gamma \varepsilon^{1-p} A,
$$

where $A$ and $B$ are as in the statement.

Finally we check whether the root $\varepsilon$ of $\varepsilon B=\varepsilon^{1-p} A$ is less than $1 / 16$; on distinguishing the cases $\varepsilon \leq 1 / 16, \varepsilon>1 / 16$ we get the inequality in (2.6).

Lemma 2.4. Let $u \in L^{\infty}\left(0, T ; \ell^{q}(V)\right)$, for a given $q>1$, be a solution of (1.1) in $V \times(0, T)$. Then for all $T>\tau_{1}>\tau_{2}>0, h \geq 0$, we have for all $0<t<T$

$$
\begin{gathered}
\sup _{\tau_{1}<\tau<t} \sum_{x \in V}(u(x, \tau)-h)_{+}^{q} d_{\omega}(x)+\int_{\tau_{1}}^{t} \sum_{x, y \in V}\left|D_{y}(u(x, \tau)-h)_{+}^{\frac{p+q-2}{p}}\right|^{p} \omega(x, y) \mathrm{d} \tau \\
\leq \frac{\gamma}{\tau_{1}-\tau_{2}} \int_{\tau_{2}}^{t} \sum_{x \in V}(u(x, \tau)-h)_{+}^{q} d_{\omega}(x) \mathrm{d} \tau . \quad(2.12)
\end{gathered}
$$

We have also, if condition (1.2) is satisfied,

$$
\begin{aligned}
\sup _{0<\tau<t} \sum_{x \in V}(u(x, \tau)-h)_{+}^{q} d_{\omega}(x) & +\int_{0}^{t} \sum_{x, y \in V}\left|D_{y}(u(x, \tau)-h)_{+}^{\frac{p+q-2}{p}}\right|^{p} \omega(x, y) \mathrm{d} \tau \\
& \leq \gamma \int_{0}^{t} \sum_{x \in V}\left(u_{0}(x)-h\right)_{+}^{q} d_{\omega}(x) \mathrm{d} \tau
\end{aligned}
$$

Proof. Let us prove (2.12); the inequality (2.13) is proved similarly.

We multiply $(1.1)$ against $\zeta(x) \eta(t)(u(x, t)-h)_{+}^{q-1}$; on integrating by parts as in the proof of Lemma 2.2 we obtain

$$
\begin{aligned}
& \frac{1}{q} \sum_{x \in V} \zeta(x)(u(x, t)-h)_{+}^{q} d_{\omega}(x) \eta(t) \\
& \quad+\int_{0}^{t} \sum_{x, y \in V}\left|D_{y} u(x, \tau)\right|^{p-2} D_{y} u(x, \tau) \zeta(y) D_{y}(u(x, \tau)-h)_{+}^{q-1} \omega(x, y) \eta(\tau) \mathrm{d} \tau \\
& \quad+\int_{0}^{t} \sum_{x, y \in V}\left|D_{y} u(x, \tau)\right|^{p-2} D_{y} u(x, \tau) D_{y} \zeta(x)(u(x, \tau)-h)_{+}^{q-1} \omega(x, y) \eta(\tau) \mathrm{d} \tau \\
& \quad=\frac{1}{q} \int_{0}^{t} \sum_{x \in V} \zeta(x)(u(x, \tau)-h)_{+}^{q} d_{\omega}(x) \eta^{\prime}(\tau) \mathrm{d} \tau .
\end{aligned}
$$


We estimate next the second integral in (2.14). The absolute value of the integrand is bounded from above by

$$
\begin{aligned}
& \frac{1}{R_{2}-R_{1}} \sum_{x, y \in B_{R_{2}+1}}\left|D_{y} u(x, \tau)\right|^{p-1}(u(x, \tau)-h)_{+}^{q-1} \omega(x, y) \\
\leq & \frac{1}{R_{2}-R_{1}} \sum_{x, y \in B_{R_{2}+1}}\left(u(x, \tau)^{p+q-2}+u(y, \tau)^{p-1} u(x, \tau)^{q-1}\right) \omega(x, y) \leq \frac{C_{u}}{R_{2}-R_{1}}
\end{aligned}
$$

where $C_{u}$ is independent of $R_{i}$. Owing to $p+q-2>q$ and to Remark 1.4, to this end it is only left to observe that

$$
\begin{aligned}
& \sum_{x, y \in B_{R_{2}+1}} u(y, \tau)^{p-1} u(x, \tau)^{q-1} \omega(x, y) \\
& \quad \leq\left(\sum_{y \in V} u(y, \tau)^{(p-1) q} d_{\omega}(y)\right)^{\frac{1}{q}}\left(\sum_{x \in V} u(x, \tau)^{q} d_{\omega}(x)\right)^{\frac{q-1}{q}},
\end{aligned}
$$

and to use once more Remark 1.4, since $(p-1) q>q$.

The sought after estimates follows immediately upon applying Lemma 2.1 with $v=0$ and then letting first $R_{2} \rightarrow \infty$ and then $R_{1} \rightarrow \infty$.

Remark 2.5. Lemma 2.4 is still in force if $u$ is the difference of two solutions to (1.1). The proof is the same, when we start from the difference of the two equations and recall Lemma 2.1.

\subsection{Existence and comparison.}

Proposition 2.6. Let $u_{0} \in \ell^{q}(V), q>1$. Then (1.1)-(1.2) has a solution in $L^{\infty}\left(0,+\infty ; \ell^{q}(V)\right)$. If $u_{0} \geq 0$ then $u \geq 0$.

Proof. Let $u_{0} \in \ell^{q}(V), q>1$. Define for $n \geq 1 u_{n}$ as the solution to

$$
\begin{aligned}
\frac{\partial u_{n}}{\partial t}(x, t) & =\Delta_{p} u_{n}(x, t), & & x \in B_{n}, t>0, \\
u_{n}(x, 0) & =u_{0}(x), & & x \in B_{n}, \\
u_{n}(x, t) & =0, & & x \notin B_{n}, t \geq 0 .
\end{aligned}
$$

In practice this is a finite system of ordinary differential equations, uniquely solvable in the class $C^{1}(0, T)$ at least as long as the solution stays bounded over $(0, T)$.

In this connection, we rewrite $(2.15),(2.17)$ as

$$
u_{n}(x, t)^{q-1} \frac{\partial u_{n}}{\partial t}(x, t)=u_{n}(x, t)^{q-1} \Delta_{p} u_{n}(x, t), \quad x \in V, t>0,
$$

where we stress that the equality holds for all $x \in V$. In this Subsection we denote $s^{q-1}=|s|^{q-1} \operatorname{sign}(s)$ for all $s \in \boldsymbol{R}$. Thus, summing over $x \in V$ and integrating by parts both in $t$ and in $x$ (in the suitable 
sense) we see that the elliptic part of the equation yields a nonnegative contribution, so that

$$
\sum_{x \in V}\left|u_{n}(x, t)\right|^{q} d_{\omega}(x) \leq \sum_{x \in B_{n}}\left|u_{0}(x)\right|^{q} d_{\omega}(x) \leq\left\|u_{0}\right\|_{\ell^{q}(V)}^{q} .
$$

In turn, as explained in Remark 1.4, this implies stable sup bounds for $u_{n}$ which, together with the discrete character of the $p$-laplacian and with the equation (2.15), also give stable sup bounds for the time derivative $\partial u_{n} / \partial t$, for each fixed $x$. However $V$ is countable, so that this is enough to enable us to extract a subsequence, still denoted by $u_{n}$ such that

$$
u_{n}(x, t) \rightarrow u(x, t), \quad \frac{\partial u_{n}}{\partial t}(x, t) \rightarrow \frac{\partial u}{\partial t}(x, t)
$$

for each $x \in V$, uniformly for $t \in[0, T]$, where we have made use of the equation again to obtain convergence for the time derivative. Finally owing to (2.18) we have

$$
\sum_{x \in V}|u(x, t)|^{q} d_{\omega}(x) \leq\left\|u_{0}\right\|_{\ell^{q}(V)}^{q}, \quad t>0 .
$$

It is easily seen that $u \in L^{\infty}\left(0,+\infty ; \ell^{q}(V)\right)$ is a solution to (1.1)-(1.2). If $u_{0} \geq 0$, we appeal to next result to prove that $u \geq 0$.

Proposition 2.7 (Comparison, [23]). If $u_{1}, u_{2} \in L^{\infty}\left(0, T ; \ell^{q}(V)\right)$ solve (1.1)-(1.2) with $u_{01}, u_{02} \in \ell^{q}(V), u_{01} \geq u_{02}$, then $u_{1} \geq u_{2}$.

2.3. Elementary inequalities. We record for future use two immediate consequences of (1.4), (1.5):

$$
\begin{aligned}
& \Lambda_{p}(s a)^{-1} \leq s^{\omega} \Lambda_{p}(a)^{-1}, \quad s \geq 1, a>0 ; \\
& \Lambda_{p}(\sigma a)^{-1} \leq \sigma^{\frac{p}{N}} \Lambda_{p}(a)^{-1}, \quad 0<\sigma \leq 1, a>0 .
\end{aligned}
$$

Also the following Lemma relies on (1.4) and will be used in a context where it is important that $\nu<1 /(p-1)$.

Lemma 2.8. Let $\nu=N(p-2) /[(N(p-2)+p)(p-1)]$ and $b>0$. Then the function

$$
\tau \mapsto \tau^{\nu} \psi_{1}^{(-1)}\left(\tau^{-1} b\right)^{\frac{p-2}{p-1}}, \quad \tau>0,
$$

is nondecreasing.

Proof. Equivalently we show that

$$
r \mapsto r^{-\alpha} \psi_{1}^{(-1)}(r)^{p-2}
$$


is nonincreasing for $\alpha=\nu(p-1)$. Set $s=\psi_{1}^{(-1)}(r)$, so that by definition of $\psi_{1}$

$$
r^{-\alpha} \psi_{1}^{(-1)}(r)^{p-2}=s^{(1-\alpha)(p-2)} \Lambda_{p}\left(s^{-1}\right)^{-\alpha}=\left[s^{-\frac{p}{N}} \Lambda_{p}\left(s^{-1}\right)\right]^{-\alpha} .
$$

By assumption (1.4) the latter quantity is indeed nonincreasing in $s$ which however is a nondecreasing function of $r$.

Lemma 2.9. Under assumption (1.7) we have that if $R, s>0, c \geq 1$ and

$$
R^{p}=\operatorname{cs} \psi_{1}^{(-1)}\left(s^{-1}\right)^{p-2},
$$

then

$$
\mu_{\omega}\left(B_{\lfloor R\rfloor}\right) \leq \gamma(c) \psi_{1}^{(-1)}\left(s^{-1}\right)^{-1} .
$$

Proof. Let $\tau>0$ be such that $s^{-1}=\psi_{1}(\tau)=\tau^{p-2} \Lambda_{p}\left(\tau^{-1}\right)$. Then

$$
c^{-1} R^{p}=\Lambda_{p}\left(\tau^{-1}\right)^{-1} \text {. }
$$

On the other hand, on setting $v=\mu_{\omega}\left(B_{\lfloor R\rfloor}\right)$ and invoking $(1.7)$ we get

$$
c^{-1} R^{p} \geq c^{-1} \mathcal{R}(v)^{p} \geq c^{-1} \gamma_{0} \Lambda_{p}(v)^{-1} \geq \Lambda_{p}\left(\left(\gamma_{0} c^{-1}\right)^{\frac{N}{p}} v\right)^{-1},
$$

where we also used (2.22). Since $\Lambda_{p}$ is nonincreasing, the result follows.

\section{Proofs of Proposition 1.6 and Corollary 1.7}

Proof of Proposition 1.6. By assumption, and by Remark $1.4, u \in L^{\infty}\left(0, T ; \ell^{q}(V)\right)$ for some $q>1$; then for all $k>0$ the cut function $(u(t)-k)_{+}$is finitely supported. For given $0<\sigma_{1}<\sigma_{2}<1 / 2, k>0,0<t<T$ define the decreasing sequences

$$
\begin{aligned}
k_{i} & =k\left[1-\sigma_{2}+2^{-i}\left(\sigma_{2}-\sigma_{1}\right)\right], & i & =0,1,2, \ldots \\
t_{i} & =\frac{t}{2}\left[1-\sigma_{2}+2^{-i}\left(\sigma_{2}-\sigma_{1}\right)\right], & i & =0,1,2, \ldots
\end{aligned}
$$

and let $f_{i}(x, \tau)=\left(u(x, \tau)-k_{i}\right)_{+}^{\nu}$, where $\nu=(p+q-2) / p$. Let also

$$
\begin{aligned}
& m_{i}(\tau)=\mu_{\omega}\left(\left\{x \in V \mid u(x, \tau)>k_{i}\right\}\right), \quad M_{i}=\sup _{t_{i}<\tau<t} m_{i}(\tau), \\
& D_{i}(\tau)=\sum_{x, y \in V}\left|D_{y} f_{i}(x, \tau)\right|^{p} \omega(x, y) .
\end{aligned}
$$

Since $b:=q / \nu<p$, it follows from Faber-Krahn inequality (1.3) and Hölder's and Young's inequalities that

$$
\begin{aligned}
\sum_{x \in V} f_{i+1}(x, \tau)^{b} d_{\omega}(x) & \leq m_{i+1}(\tau)^{1-\frac{b}{p}} \Lambda_{p}\left(m_{i+1}(\tau)\right)^{-\frac{b}{p}} D_{i+1}(\tau)^{\frac{b}{p}} \\
\leq & \varepsilon^{\frac{p}{b}} D_{i+1}(\tau)+\varepsilon^{-\frac{p}{p-b}} \Lambda_{p}\left(m_{i+1}(\tau)\right)^{-\frac{b}{p-b}} m_{i+1}(\tau) .
\end{aligned}
$$


Here $\varepsilon>0$ is arbitrary and will be selected below.

We integrate $(3.1)$ over $\left(t_{i+1}, t\right)$ to find

$$
\begin{aligned}
& \int_{t_{i+1}}^{t} \sum_{x \in V} f_{i+1}(x, \tau)^{b} d_{\omega}(x) \mathrm{d} \tau \leq \varepsilon^{\frac{p}{b}} \int_{t_{i+1}}^{t} D_{i+1}(\tau) \mathrm{d} \tau \\
& \quad+\varepsilon^{-\frac{p}{p-b}} \int_{t_{i+1}}^{t} \Lambda_{p}\left(m_{i+1}(\tau)\right)^{-\frac{b}{p-b}} m_{i+1}(\tau) \mathrm{d} \tau \\
& \leq \varepsilon^{\frac{p}{b}} \int_{t_{i+1}}^{t} D_{i+1}(\tau) \mathrm{d} \tau+\varepsilon^{-\frac{p}{p-b}} t \Lambda_{p}\left(M_{i+1}\right)^{-\frac{b}{p-b}} M_{i+1} .
\end{aligned}
$$

Next we invoke Lemma 2.4 with $\tau_{1}=t_{i}, \tau_{2}=t_{i+1}, h=k_{i}$, to infer

$$
\begin{aligned}
L_{i} & :=\sup _{t_{i}<\tau<t} \sum_{x \in V} f_{i}(x, \tau)^{b} d_{\omega}(x)+\int_{t_{i}}^{t} D_{i}(\tau) \mathrm{d} \tau \\
\leq & \frac{\gamma 2^{i}}{t\left(\sigma_{2}-\sigma_{1}\right)} \int_{t_{i+1}}^{t} \sum_{x \in V} f_{i+1}(x, \tau)^{b} d_{\omega}(x) \mathrm{d} \tau \\
\leq & \frac{\gamma 2^{i}}{t\left(\sigma_{2}-\sigma_{1}\right)} \varepsilon^{\frac{p}{b}} \int_{t_{i+1}}^{t} D_{i+1}(\tau) \mathrm{d} \tau \\
& +\frac{\gamma 2^{i}}{\sigma_{2}-\sigma_{1}} \varepsilon^{-\frac{p}{p-b}} \Lambda_{p}\left(M_{i+1}\right)^{-\frac{b}{p-b}} M_{i+1},
\end{aligned}
$$

where the second inequality follows of course from (3.2). For a $\delta>0$ to be chosen, select ( $\gamma$ denotes here the constant in (3.3))

$$
\frac{\gamma 2^{i}}{t\left(\sigma_{2}-\sigma_{1}\right)} \varepsilon^{\frac{p}{b}}=\delta \quad \text { i.e., } \quad \varepsilon=\gamma_{0} \delta^{\frac{b}{p}} t^{\frac{b}{p}}\left(\sigma_{2}-\sigma_{1}\right)^{\frac{b}{p}} 2^{-\frac{b}{p} i}
$$

On substituting this choice of $\varepsilon$ in (3.3) we arrive at an estimate which can be successfully iterated, that is

$$
L_{i} \leq \delta L_{i+1}+\frac{\gamma 2^{\frac{p i}{p-b}}}{\left(\sigma_{2}-\sigma_{1}\right)^{\frac{p}{p-b}}} \delta^{-\frac{b}{p-b}} t^{-\frac{b}{p-b}} \Lambda_{p}\left(M_{\infty}\right)^{-\frac{b}{p-b}} M_{\infty}
$$

Here we set

$$
\begin{gathered}
t_{\infty}=\lim _{i \rightarrow \infty} t_{i}=\frac{t}{2}\left(1-\sigma_{2}\right), \quad k_{\infty}=\lim _{i \rightarrow \infty} k_{i}=k\left(1-\sigma_{2}\right), \\
M_{\infty}=\sup _{t_{\infty}<\tau<t} \mu_{\omega}\left(\left\{x \in V \mid u(x, \tau)>k_{\infty}\right\}\right) .
\end{gathered}
$$


On iterating (3.4) we infer

$$
L_{0} \leq \delta^{j} L_{j}+\left(\sum_{i=0}^{j} \delta^{i} 2^{\frac{p i}{p-b}}\right) \frac{\gamma}{\left(\sigma_{2}-\sigma_{1}\right)^{\frac{p}{p-b}}} t^{-\frac{b}{p-b}} \Lambda_{p}\left(M_{\infty}\right)^{-\frac{b}{p-b}} M_{\infty},
$$

which yields as $j \rightarrow \infty$, provided we select $\delta<2^{-p /(p-b)}$,

$$
\begin{aligned}
& \sup _{t\left(1-\sigma_{1}\right) / 2<\tau<t} \sum_{x \in V}\left(u(x, \tau)-k\left(1-\sigma_{1}\right)\right)_{+}^{q} d_{\omega}(x) \leq L_{0} \\
& \leq \frac{\gamma}{\left(\sigma_{2}-\sigma_{1}\right)^{\frac{q}{p-2}}} t^{-\frac{q}{p-2}} \Lambda_{p}\left(M_{\infty}\right)^{-\frac{q}{p-2}} M_{\infty},
\end{aligned}
$$

for $M_{\infty}$ as in (3.6), owing also to $b /(p-b)=q /(p-2)$.

The proof will be concluded by a second process of iteration, built on (3.7). Let $1 / 2>\sigma>0$ and $k>0$, and define the increasing sequences

$$
\begin{gathered}
\tau_{n}=\frac{t}{2}\left(1-\sigma 2^{-n}\right), \quad h_{n}=k\left(1-\sigma 2^{-n}\right), \\
\bar{h}_{n}=\frac{h_{n}+h_{n+1}}{2}=k\left(1-3 \sigma 2^{-n-2}\right), \quad n \geq 0,
\end{gathered}
$$

as well as the decreasing one

$$
Y_{n}=\sup _{\tau_{n}<\tau<t} \mu_{\omega}\left(\left\{x \in V \mid u(x, \tau)>h_{n}\right\}\right) .
$$

Next we apply Chebychev's inequality to find

$$
Y_{n+1} \leq 2^{(n+2) q} \sigma^{-q} k^{-q} \sup _{\tau_{n+1}<\tau<t} \sum_{x \in V}\left(u(x, \tau)-\bar{h}_{n}\right)_{+} d_{\omega}(x) .
$$

The right hand side of (3.8) is then majorized by appealing to (3.7), where we select

$$
\sigma_{1}=3 \sigma 2^{-n-2}, \quad \sigma_{2}=\sigma 2^{-n}
$$

in order to obtain

$$
Y_{n+1} \leq \gamma \sigma^{-\frac{q(p-1)}{p-2}} 2^{\frac{n(p-2+q)}{p-2}} t^{-\frac{q}{p-2}} k^{-q} \Lambda_{p}\left(Y_{n}\right)^{-\frac{q}{p-2}} Y_{n}
$$

In turn, on invoking our assumption (1.4), we transform (3.9) into

$$
Y_{n+1} \leq \gamma \sigma^{-\frac{q(p-1)}{p-2}} 2^{\frac{n(p-2+q)}{p-2}} t^{-\frac{q}{p-2}} k^{-q} \Lambda_{p}\left(Y_{0}\right)^{-\frac{q}{p-2}} Y_{0}^{-\frac{p}{N} \frac{q}{p-2}} Y_{n}^{1+\frac{p}{N} \frac{q}{p-2}} .
$$

This inequality yields $Y_{n} \rightarrow 0$ as $n \rightarrow \infty$ provided we choose $k$ so that (see [21, Lemma 5.6 Ch. II])

$$
k^{-1} t^{-\frac{1}{p-2}} \Lambda_{p}\left(Y_{0}\right)^{-\frac{1}{p-2}} \leq \gamma_{0}(q, p, N) .
$$

In this connection we may assume e.g., $\sigma=1 / 4$. The proof is concluded when we remark that $Y_{n} \rightarrow 0$ immediately implies

$$
u(x, t) \leq k, \quad x \in V .
$$


Remark 3.1. We note that the proof of Proposition 1.6 makes use of the differential equation (1.1) only thru inequality (2.12). This fact will be used below.

Proof of Corollary 1.7. We remark on using Chebychev's inequality once more that in (3.11)

$$
Y_{0} \leq 2^{r} k^{-r} \sup _{\frac{t}{4}<\tau<t} \sum_{x \in V} u(x, \tau)^{r} d_{\omega}(x) .
$$

Let us set

$$
E_{r}=\sup _{0<\tau<t} \sum_{x \in V} u(x, \tau)^{r} d_{\omega}(x) .
$$

Then (3.11) is certainly fulfilled if

$$
k^{-1} t^{-\frac{1}{p-2}} \Lambda_{p}\left(k^{-r} E_{r}\right)^{-\frac{1}{p-2}}=\gamma_{0},
$$

where we also used (2.21). On the other hand, if we set

$$
\psi_{r}(s)=s^{\frac{p-2}{r}} \Lambda_{p}\left(s^{-1}\right), \quad s>0,
$$

then (3.12) amounts to

$$
k=E_{r}^{\frac{1}{r}}\left[\psi_{r}^{(-1)}\left(\gamma t^{-1} E_{r}^{-\frac{p-2}{r}}\right)\right]^{\frac{1}{r}} \leq \gamma E_{r}^{\frac{1}{r}}\left[\psi_{r}^{(-1)}\left(t^{-1} E_{r}^{-\frac{p-2}{r}}\right)\right]^{\frac{1}{r}},
$$

where we have made use of the definition of $\psi_{r}$ and of $(2.22)$.

\section{Proof of Theorem 1.9}

Let $u_{0} \in \ell^{1}(V), u_{0} \geq 0$. Then we have also $u_{0} \in \ell^{2}(V)$ as noted in Remark 1.4, and we may consider the solution $u \geq 0$ constructed according to Subsection 2.2. First we bound the $\ell^{1}(V)$ norm of the solution from above, that is we prove estimate (4.1) below. Actually, this estimate follows from the contractivity result in [23], but we present an argument for it here in order to make this proof more self-contained. We multiply the equation against $\Theta(u(x, \tau)) \zeta(x)$ where $\zeta$ is as in Section 2 ,

$$
\Theta(u)=\frac{(u-h)_{+}}{u+\varepsilon}
$$

for any given $h>0, \varepsilon>0$, and integrate by parts. The purpose of the cut at level $h$ is to ease technically the argument. Since $\Theta$ is a 
nondecreasing function, reasoning as in the proof of Lemma 2.4 we easily obtain

$$
\begin{array}{r}
\sum_{x \in V} \int_{0}^{u(x, t)} \frac{(s-h)_{+}}{s+\varepsilon} \zeta(x) d_{\omega}(x) \leq \sum_{x \in V} \int_{0}^{u_{0}(x)} \frac{(s-h)_{+}}{s+\varepsilon} d_{\omega}(x)+K_{1} \\
\leq \sum_{x \in V} u_{0}(x) d_{\omega}(x)+K_{1},
\end{array}
$$

where

$$
\begin{aligned}
& K_{1}=\frac{1}{R_{2}-R_{1}} \int_{0}^{t} \sum_{x \in V}\left|D_{y} u(x, \tau)\right|^{p-1} \chi_{\{u(\tau)>h\}}(x) \omega(x, y) \mathrm{d} \tau \\
& \quad \leq \frac{h^{-1}}{R_{2}-R_{1}} \int_{0}^{t} \sum_{x \in V}\left|D_{y} u(x, \tau)\right|^{p-1} u(x, \tau) \omega(x, y) \mathrm{d} \tau .
\end{aligned}
$$

Then we may proceed as in the proof of Lemma 2.4 with $q=2$ and let $R_{2} \rightarrow \infty$ and then $R_{1} \rightarrow \infty$ to make $K_{1}$ vanish. Finally we let first $\varepsilon \rightarrow 0$ and then $h \rightarrow 0$ : on invoking the monotone convergence theorem we get

$$
\|u(t)\|_{\ell^{1}(V)} \leq\left\|u_{0}\right\|_{\ell^{1}(V)} .
$$

Therefore from Corollary 1.7 and Remark 1.8 we infer that (1.15) is satisfied.

In order to prove (1.14) we proceed as follows. We multiply the equation against $\zeta(x)$ as above and integrate by parts obtaining

$$
\sum_{x \in V} u(x, t) \zeta(x) d_{\omega}(x)+K_{2}=\sum_{x \in V} u_{0}(x) \zeta(x) d_{\omega}(x),
$$

where

$$
\begin{aligned}
\left|K_{2}\right| & =\left.\left|\int_{0}^{t} \sum_{x \in V}\right| D_{y} u(x, \tau)\right|^{p-2} D_{y} u(x, \tau) D_{y} \zeta(x) \omega(x, y) \mathrm{d} \tau \mid \\
& \leq \frac{1}{R_{2}-R_{1}} \int_{0}^{t} \sum_{x \in B_{R_{2}}+1}\left|D_{y} u(x, \tau)\right|^{p-1} \omega(x, y) \mathrm{d} \tau \\
& \leq \frac{2 t}{\left(R_{2}-R_{1}\right) \Lambda_{p}^{(-1)}(2)^{p-2}}\left\|u_{0}\right\|_{\ell^{1}(V)}^{p-1} .
\end{aligned}
$$

Here we reasoned as in (1.10) (with $q=1$ ), exploiting $p>2$ and the already proved bound (4.1). Then we rewrite (4.2) as

$$
\|u(t)\|_{\ell^{1}(V)}+K_{2} \geq \sum_{x \in B_{R_{1}}} u_{0}(x) d_{\omega}(x)
$$


and let first $R_{2} \rightarrow \infty$ then $R_{1} \rightarrow \infty$ to obtain the converse to (4.1).

Finally we prove the entropy estimate (1.16). First we invoke Hölder's inequality to bound

$$
\begin{aligned}
I:= & \int_{0}^{t} \sum_{x, y \in V}\left|D_{y} u(x, \tau)\right|^{p-1} \omega(x, y) \mathrm{d} \tau \\
\leq & \left(\int_{0}^{t} \sum_{x, y \in V} \tau^{-\delta(p-1)}(u(x, \tau)+u(y, \tau))^{(2-\theta)(p-1)} \omega(x, y) \mathrm{d} \tau\right)^{\frac{1}{p}} \\
& \times\left(\int_{0}^{t} \sum_{x, y \in V} \tau^{\delta}\left|D_{y} u(x, \tau)\right|^{p}(u(x, \tau)+u(y, \tau))^{\theta-2} \omega(x, y) \mathrm{d} \tau\right)^{\frac{p-1}{p}} \\
= & : K_{3}^{\frac{1}{p}} K_{4}^{\frac{p-1}{p}} .
\end{aligned}
$$

Here $\delta>0$ is to be chosen and we select

$$
\theta=\frac{2 p-3}{p-1} \in(1,2), \quad \text { so that } \quad(2-\theta)(p-1)=1 .
$$

Thus

$$
K_{3} \leq 2 \int_{0}^{t} \tau^{-\delta(p-1)}\|u(\tau)\|_{\ell^{1}(V)} \mathrm{d} \tau \leq \gamma\left\|u_{0}\right\|_{\ell^{1}(V)} t^{1-\delta(p-1)},
$$

provided

$$
\delta(p-1)<1
$$

In order to bound $K_{4}$ we multiply the differential equation against $\tau^{\delta} u^{\theta-1}$ and integrate by parts. After dropping a positive contribution from the left hand side of the resulting equality we obtain

$$
\begin{aligned}
K_{4} & \leq \gamma \int_{0}^{t} \sum_{x \in V} \tau^{\delta-1} u(x, \tau)^{\frac{p-2}{p-1}+1} d_{\omega}(x) \mathrm{d} \tau \\
& \leq \gamma\left\|u_{0}\right\|_{\ell^{1}(V)} \int_{0}^{t} \tau^{\delta-1}\|u(\tau)\|_{\ell^{\infty}(V)}^{\frac{p-2}{p-1}} \mathrm{~d} \tau \\
& \leq \gamma\left\|u_{0}\right\|_{\ell^{1}(V)}^{\frac{p-2}{p-1}+1} \int_{0}^{t} \tau^{\delta-1} \psi_{1}^{(-1)}\left(\tau^{-1}\left\|u_{0}\right\|_{\ell^{1}(V)}^{2-p}\right)^{\frac{p-2}{p-1}} \mathrm{~d} \tau .
\end{aligned}
$$


Select now $\nu<\delta<1 /(p-1)$, where $\nu$ is the constant defined in Lemma 2.8. Accordingly, the last integral above is bounded by

$$
\begin{aligned}
\int_{0}^{t} \tau^{\delta-\nu-1} \tau^{\nu} \psi_{1}^{(-1)}\left(\tau^{-1} \|\right. & \left.u_{0} \|_{\ell^{1}(V)}^{2-p}\right)^{\frac{p-2}{p-1}} \mathrm{~d} \tau \\
& \leq t^{\nu} \psi_{1}^{(-1)}\left(t^{-1}\left\|u_{0}\right\|_{\ell^{1}(V)}^{2-p}\right)^{\frac{p-2}{p-1}}(\delta-\nu)^{-1} t^{\delta-\nu}
\end{aligned}
$$

Collecting all the estimates in (4.4)-(4.8), we finally arrive at

$$
I \leq \gamma t^{\frac{1}{p}}\left\|u_{0}\right\|_{\ell^{1}(V)}^{\frac{2(p-1)}{p}} \psi_{1}^{(-1)}\left(t^{-1}\left\|u_{0}\right\|_{\ell^{1}(V)}^{2-p}\right)^{\frac{p-2}{p}} .
$$

\section{Proof of Theorem 1.11 And Corollary 1.12}

Proof of Theorem 1.11. Let $u$ be as in the statement of Theorem 1.11. For all $t>0, R \in \boldsymbol{N}$ we write

$$
\|u(t)\|_{\ell^{1}(V)}=\|u(t)\|_{\ell^{1}\left(B_{R}\right)}+\|u(t)\|_{\ell^{1}\left(V \backslash B_{R}\right)} .
$$

Here we denote for a fixed $x_{0} \in V$

$$
B_{R}=B_{R}\left(x_{0}\right), \quad|x|=d\left(x, x_{0}\right), \quad x \in V .
$$

For the sake of clarity let us denote by $\zeta_{R_{1}, R_{2}}$ the cutoff function defined in Section 2. Let $\rho>4 R, R \geq 4, \rho, R \in \boldsymbol{N}$ and $\phi=1-\zeta_{R, 2 R}$. We use $|x|^{\alpha} \phi(x) \zeta_{\rho, 2 \rho}(x)$ as a testing function in (1.1), for a fixed $0<\alpha<1$. We obtain, assuming in addition that $R$ is so large as $u_{0}(x)=0$ for $x \notin B_{R}$,

$$
\begin{aligned}
& \sum_{x \in V}|x|^{\alpha} \phi(x) \zeta_{\rho, 2 \rho}(x) u(x, t) d_{\omega}(x) \\
& =-\int_{0}^{t} \sum_{x \in V}\left|D_{y} u(x, \tau)\right|^{p-2} D_{y} u(x, \tau) D_{y}\left[\phi(x) \zeta_{\rho, 2 \rho}(x)|x|^{\alpha}\right] \omega(x, y) \mathrm{d} \tau .
\end{aligned}
$$

In last integral, the term originating from $D_{y} \zeta_{\rho, 2 \rho}$ is seen to become vanishingly small as $\rho \rightarrow \infty$, since $\alpha<1$, similarly to what we did to 
bound $K_{2}$ in Section 4 . Thus in the limit $\rho \rightarrow \infty$ we get

$$
\begin{aligned}
& \sum_{x \notin B_{2 R}}|x|^{\alpha} u(x, t) d_{\omega}(x) \\
& \leq-\int_{0}^{t} \sum_{x \in V}\left|D_{y} u(x, \tau)\right|^{p-2} D_{y} u(x, \tau) D_{y}\left[\phi(x)|x|^{\alpha}\right] \omega(x, y) \mathrm{d} \tau \\
& =\int_{0}^{t} \sum_{x \in V}\left|D_{y} u(x, \tau)\right|^{p-2} D_{y} u(x, \tau) D_{y} \zeta_{R, 2 R}(x)|y|^{\alpha} \omega(x, y) \mathrm{d} \tau \\
& \quad-\int_{0}^{t} \sum_{x \in V}\left|D_{y} u(x, \tau)\right|^{p-2} D_{y} u(x, \tau) D_{y}|x|^{\alpha} \phi(x) \omega(x, y) \mathrm{d} \tau=: Q_{1}+Q_{2} .
\end{aligned}
$$

Since if $x \sim y, x \notin B_{R}$,

$$
\left.\left|D_{y}\right| x\right|^{\alpha} \mid \leq \alpha \min (|x|,|y|)^{\alpha-1} \leq \gamma R^{\alpha-1},
$$

we have

$$
\left|Q_{1}\right|+\left|Q_{2}\right| \leq \gamma R^{\alpha-1} \int_{0}^{t} \sum_{x \in V}\left|D_{y} u(x, \tau)\right|^{p-1} \omega(x, y) \mathrm{d} \tau .
$$

We bound the last integral by means of (1.16), concluding as follows:

$$
\begin{aligned}
& \sum_{x \notin B_{2 R}} u(x, t) d_{\omega}(x) \leq R^{-\alpha} \sum_{x \notin B_{2 R}}|x|^{\alpha} u(x, t) d_{\omega}(x) \\
& \leq \gamma R^{-1} t^{\frac{1}{p}}\left\|u_{0}\right\|_{\ell^{1}(V)}^{\frac{2(p-1)}{p}} \psi_{1}^{(-1)}\left(t^{-1}\left\|u_{0}\right\|_{\ell^{1}(V)}^{-(p-2)}\right)^{\frac{p-2}{p}} \leq \gamma \Gamma^{-1}\left\|u_{0}\right\|_{\ell^{1}(V)},
\end{aligned}
$$

where we have selected

$$
R \geq R_{p}\left(u_{0}, t\right):=\Gamma t^{\frac{1}{p}}\left\|u_{0}\right\|_{\ell^{1}(V)}^{\frac{p-2}{p}} \psi_{1}^{(-1)}\left(t^{-1}\left\|u_{0}\right\|_{\ell^{1}(V)}^{-(p-2)}\right)^{\frac{p-2}{p}},
$$

for a $\Gamma>0$. This together with conservation of mass (1.14) proves (1.17), upon an unessential redefinition of $R$.

In order to prove (1.19) we remark that from the argument above it follows that for $R$ as in (5.2),

$$
\begin{aligned}
\sum_{x \in V}|x|^{\alpha} u(x, t) d_{\omega}(x) \leq(2 R)^{\alpha} & \sum_{x \in B_{2 R}} u(x, t) d_{\omega}(x) \\
& +\sum_{x \notin B_{2 R}}|x|^{\alpha} u(x, t) d_{\omega}(x) \leq \gamma R^{\alpha}\left\|u_{0}\right\|_{\ell^{1}(V)},
\end{aligned}
$$

where we have used conservation of mass again. 
Proof of Corollary 1.12. For a suitable choice of $\Gamma$, setting $R=2 R_{p}$, $R_{p}$ as in (5.2), we have from (5.1)

$$
\|u(t)\|_{\ell^{\infty}(V)} \mu_{\omega}\left(B_{R}(t)\right) \geq\|u(t)\|_{\ell^{1}\left(B_{R}(t)\right)} \geq \frac{1}{2}\left\|u_{0}\right\|_{\ell^{1}(V)} .
$$

The statement in (1.20) then follows, if (1.7) is assumed, on invoking Lemma 2.9.

\section{Proof of Theorem 1.13}

We follow here ideas from [3], [4], [1]. Let $u_{R}$ be the solution to (1.1) with initial data

$$
u_{R}(x, 0)=u_{0}(x) \chi_{B_{R}\left(x_{0}\right)}(x), \quad x \in V .
$$

Then mass conservation and (1.13) with $r=1$ imply

$$
\left\|u_{R}(t)\right\|_{\ell \infty(V)} \leq \gamma m_{R} \psi_{1}^{(-1)}\left(t^{-1} m_{R}^{-(p-2)}\right), \quad t>0,
$$

where

$$
m_{R}=\sum_{x \in B_{R}\left(x_{0}\right)} u_{0}(x) d_{\omega}(x) .
$$

Let us also define $w_{R}=u-u_{R}$; note that $w_{R} \geq 0$ by Proposition 2.7. In spite of the fact that $w_{R}$ does not solve (1.1) we may still prove the following inequality for $h \geq 0, t>\tau_{1}>\tau_{2}>0$, also by appealing to Lemma 2.1:

$$
\begin{gathered}
\sup _{\tau_{1}<\tau<t} \sum_{x \in V}\left(w_{R}(x, \tau)-h\right)_{+}^{q} d_{\omega}(x)+\int_{\tau_{1}}^{t} \sum_{x, y \in V}\left|D_{y}\left(w_{R}(x, \tau)-h\right)_{+}^{\frac{p+q-2}{p}}\right|^{p} \omega(x, y) \mathrm{d} \tau \\
\leq \frac{\gamma}{\tau_{1}-\tau_{2}} \int_{\tau_{2}}^{t} \sum_{x \in V}\left(w_{R}(x, \tau)-h\right)_{+}^{q} d_{\omega}(x) \mathrm{d} \tau .
\end{gathered}
$$

As already observed in Remark 3.1 this is enough for us to apply Proposition 1.6 and thus Corollary 1.7 to $w_{R}$, and get

$$
\left\|w_{R}\right\|_{\ell \infty(V)} \leq \gamma E_{q}^{\frac{1}{q}}\left[\psi_{q}^{(-1)}\left(t^{-1} E_{q}^{-\frac{p-2}{q}}\right)\right]^{\frac{1}{q}} \leq \gamma E_{q 0}^{\frac{1}{q}}\left[\psi_{q}^{(-1)}\left(t^{-1} E_{q 0}^{-\frac{p-2}{q}}\right)\right]^{\frac{1}{q}},
$$

where by invoking a simple variant of $(6.2)$ with $h=0$ we find

$$
E_{q}:=\sup _{0<\tau<t} \sum_{x \in V} w_{R}(x, \tau)^{q} \leq E_{q 0}:=\sum_{x \notin B_{R}\left(x_{0}\right)} u_{0}(x)^{q} d_{\omega}(x) .
$$

We use here also Remark 1.8. 
Thus we have that for all $R>0$, since $u=u_{R}+w_{R}$,

$$
\|u(t)\|_{\ell^{\infty}(V)} \leq \gamma\left\{m_{R} \psi_{1}^{(-1)}\left(t^{-1} m_{R}^{-(p-2)}\right)+E_{q 0}^{\frac{1}{q}}\left[\psi_{q}^{(-1)}\left(t^{-1} E_{q 0}^{-\frac{p-2}{q}}\right)\right]^{\frac{1}{q}}\right\} .
$$

The first term on the right hand side of (6.4) is increasing in $R$, while the second one is decreasing. We aim at making them equal, but this is in general impossible in the discrete setting of graphs. We instead select $R$ as any number (optimally the minimum one) such that

$$
m_{R} \psi_{1}^{(-1)}\left(t^{-1} m_{R}^{-(p-2)}\right) \geq E_{q 0}^{\frac{1}{q}}\left[\psi_{q}^{(-1)}\left(t^{-1} E_{q 0}^{-\frac{p-2}{q}}\right)\right]^{\frac{1}{q}} .
$$

Then (1.22) is proved under assumption (6.5).

We need to make (6.5) explicit. First, we define

$$
X_{1}=\psi_{1}^{(-1)}\left(t^{-1} m_{R}^{-(p-2)}\right), \quad X_{q}=\psi_{q}^{(-1)}\left(t^{-1} E_{q 0}^{-\frac{p-2}{q}}\right),
$$

so that from the definition of $\psi_{r}$, we get

$$
X_{1}=t^{-\frac{1}{p-2}} m_{R}^{-1} \Lambda_{p}\left(X_{1}^{-1}\right)^{-\frac{1}{p-2}}, \quad X_{q}^{\frac{1}{q}}=t^{-\frac{1}{p-2}} E_{q 0}^{-\frac{1}{q}} \Lambda_{p}\left(X_{q}^{-1}\right)^{-\frac{1}{p-2}} .
$$

Therefore (6.5) can be written as

$$
\Lambda_{p}\left(X_{1}^{-1}\right) \leq \Lambda_{p}\left(X_{q}^{-1}\right), \quad \text { that is } \quad X_{1} \leq X_{q} .
$$

We apply $\psi_{1}$ and write the last inequality in the form

$$
\begin{aligned}
\left(t m_{R}^{p-2}\right)^{-1} & \leq \psi_{1}\left(X_{q}\right)=X_{q}^{p-2} \Lambda_{p}\left(X_{q}^{-1}\right) \\
& =X_{q}^{\frac{(p-2)(q-1)}{q}} \psi_{q}\left(X_{q}\right)=X_{q}^{\frac{(p-2)(q-1)}{q}}\left(t E_{q 0}^{\frac{p-2}{q}}\right)^{-1} .
\end{aligned}
$$

From here we immediately get, on recalling the definition of $\psi_{q}$,

$$
\frac{1}{t} \geq\left[\frac{E_{q 0}}{m_{R}}\right]^{\frac{p-2}{q-1}} \Lambda_{p}\left(m_{R}^{\frac{q}{q-1}} E_{q 0}^{-\frac{1}{q-1}}\right) .
$$

This amounts to (1.23) concluding the proof.

\section{REFERENCES}

[1] N. V. Afanas' eva and A. F. Tedeev. Fujita-type theorems for quasilinear parabolic equations in the case of slowly vanishing initial data. Mat. Sb., 195(4):322, 2004.

[2] S. Andres, M. T. Barlow, J.-D. Deuschel, and B. M. Hambly. Invariance principle for the random conductance model. Probab. Theory Related Fields, 156(34):535-580, 2013.

[3] D. Andreucci. Degenerate parabolic equations with initial data measures. Transactions American Mathematical Society, 349:3911-3923, 1997. American Mathematical Society. 
[4] D. Andreucci, R. Cirmi, S. Leonardi, and A. F. Tedeev. Large time behavior of solutions to the Neumann problem for a quasilinear second order degenerate parabolic equation in domains with noncompact boundary. Journal of Differential Equations, 174:253-288, 2001. Elsevier.

[5] D. Andreucci and A. F. Tedeev. A Fujita type result for a degenerate Neumann problem in domains with non compact boundary. J. Math. Analysis and Appl., 231:543-567, 1999. Elsevier.

[6] D. Andreucci and A. F. Tedeev. Sharp estimates and finite speed of propagation for a Neumann problem in domains narrowing at infinity. Advances Diff. Eqs., 5:833-860, 2000. Khayyam Publ., Athens Ohio (U.S.A.).

[7] D. Andreucci and A. F. Tedeev. Optimal decay rate for degenerate parabolic equations on noncompact manifolds. Methods Appl. Anal., 22(4):359-376, 2015.

[8] D. Bakry, T. Coulhon, M. Ledoux, and L. Saloff-Coste. Sobolev inequalities in disguise. Indiana Univ. Math. J., 44(4):1033-1074, 1995.

[9] M. Barlow, T. Coulhon, and A. Grigor'yan. Manifolds and graphs with slow heat kernel decay. Invent. Math., 144(3):609-649, 2001.

[10] M. Bonforte and G. Grillo. Singular evolution on manifolds, their smoothing properties, and Sobolev inequalities. Discrete Contin. Dyn. Syst., (Dynamical Systems and Differential Equations. Proceedings of the 6th AIMS International Conference, suppl.):130-137, 2007.

[11] F. R. K. Chung. Spectral graph theory, volume 92 of CBMS Regional Conference Series in Mathematics. Published for the Conference Board of the Mathematical Sciences, Washington, DC; by the American Mathematical Society, Providence, RI, 1997.

[12] S.-Y. Chung and M.-J. Choi. Blow-up solutions and global solutions to discrete p-Laplacian parabolic equations. Abstr. Appl. Anal., pages Art. ID 351675, 11, 2014.

[13] S.-Y. Chung and J.-H. Park. A complete characterization of extinction versus positivity of solutions to a parabolic problem of $p$-Laplacian type in graphs. $J$. Math. Anal. Appl., 452(1):226-245, 2017.

[14] T. Coulhon and A. Grigoryan. Random walks on graphs with regular volume growth. Geom. Funct. Anal., 8(4):656-701, 1998.

[15] E. DiBenedetto. Degenerate Parabolic Equations. Springer-Verlag, New York, NY, 1993.

[16] E. DiBenedetto and M. A. Herrero. On the Cauchy problem and initial traces for a degenerate parabolic equation. Transactions of American Mathematical Society, 314:187-224, 1989.

[17] A. Elmoataz, M. Toutain, and D. Tenbrinck. On the $p$-Laplacian and $\infty$ Laplacian on graphs with applications in image and data processing. SIAM J. Imaging Sci., 8(4):2412-2451, 2015.

[18] A. Grigor'yan. Analysis on Graphs. Lecture Notes. University of Bielefeld, 2009.

[19] B. Hua and D. Mugnolo. Time regularity and long-time behavior of parabolic p-Laplace equations on infinite graphs. J. Differential Equations, 259(11):61626190, 2015.

[20] M. Keller and D. Mugnolo. General Cheeger inequalities for $p$-Laplacians on graphs. Nonlinear Anal., 147:80-95, 2016. 
[21] O. A. Ladyzhenskaja, V. A. Solonnikov, and N. N. Ural'ceva. Linear and Quasilinear Equations of Parabolic Type, volume 23 of Translations of Mathematical Monographs. American Mathematical Society, Providence, RI, 1968.

[22] Y. Lin and Y. Wu. The existence and nonexistence of global solutions for a semilinear heat equation on graphs. Calc. Var. Partial Differential Equations, 56(4):Art. 102, 22, 2017.

[23] D. Mugnolo. Parabolic theory of the discrete $p$-Laplace operator. Nonlinear Anal., 87:33-60, 2013.

[24] M. I. Ostrovskii. Sobolev spaces on graphs. Quaest. Math., 28(4):501-523, 2005.

[25] A. F. Tedeev. Estimates for the rate of stabilization as $t \rightarrow \infty$ of the solution of the second mixed problem for a second-order quasilinear parabolic equation. Differentsial' nye Uravneniya, 27(10):1795-1806, 1838, 1991.

[26] D. L. Wang and P. Wang. Discrete isoperimetric problems. SIAM J. Appl. Math., 32(4):860-870, 1977.

Department of Basic and Applied Sciences for Engineering, Sapienza UniVERSiTy of Rome, ITALY

Email address: daniele.andreucci@sbai.uniroma1.it

South Mathematical Institute of VSC RAS, Vladikavkaz, Russian FEDERATION

Email address: a_tedeev@yahoo.com 